\title{
INVESTIGATION OF MR FLUIDS IN THE OSCILLATORY SQUEEZE MODE
}

\author{
Bogdan SAPIŃSKI*, Wojciech HORAK**, Marcin SZCZĘCH ${ }^{* *}$
}

\author{
*AGH University of Science and Technology, Faculty of Mechanical Engineering and Robotics, Department of Process Control, \\ Al. Mickiewicza 30, 30-059 Kraków, Poland \\ *AGH University of Science and Technology Faculty of Mechanical Engineering and Robotics, Department of Machine Design and Technology, \\ Al. Mickiewicza 30, 30-059 Kraków, Poland \\ deep@agh.edu.pl, horak@agh.edu.pl, szczech@agh.edu.pl
}

\begin{abstract}
The paper summarises the results of laboratory testing of three commercially available magnetorheological (MR) fluids operated in the oscillatory squeeze mode. Tested fluids include the Basonetic 204 and Basonetic 4035 (BASF) and MRF-122EG (Lord Corporation). The oscillatory squeeze mode produces large forces at small displacements. This feature may be well utilised in fabrication of new MR devices. The purpose of the experiments was to evaluate the suitability of MR fluids for applications in MR vibration dampers being developed under the current research project. The results enable a comparative analysis of investigated fluids and verification of phenomena encountered in the oscillatory squeeze mode and reported in the literature.
\end{abstract}

Key words: MR Fluid, Oscillatory Squeeze Mode, Experiments

\section{INTRODUCTION}

MR fluids are suspensions of ferromagnetic particles in a carrier liquid (typically oil or water) whose rheological properties can be controlled through the action of a magnetic field. The fluids can be operated in the valve mode (used in vibration dampers (Sapiński, 2006)), in the direct shear mode (used in brakes and couplings) or in the squeeze mode (used in dampers and bearings (Bonneau and Frene, 1997; Farjoud et al., 2008; Guldbakke and Hesselbach, 2006; Mazlan, 2008)). Literature on the subjects abounds in reports on the valve and coupling modes whilst the squeeze mode still poses a major challenge to researchers.

Properties of MR fluids operated in the squeeze mode are dependent on application requirements. In the case of vibration damping systems, of particular importance is the ability to generate significant forces when MR fluid is displaced from the squeeze zone and to provide for a wide variability range of rheological parameters.

This paper summarises the laboratory testing of selected MR fluids operated in the oscillatory squeeze mode with a constant contact area between the sample and plate. Since MR fluids dedicated directly to this operation mode are not available commercially, experiments were done on samples of MR fluids which, according to the manufacturers' specifications, are recommended for use in vibration dampers.

The purpose of the experiments was to evaluate the suitability of those MR fluids for use in squeeze mode vibration dampers, which are investigated by the authors under the current research project.

\section{SQUEEZE MOEDE}

Two cases of the squeeze mode operation of MR fluids are considered: with the constant volume (Fig. 1a) and with the constant contact area (Fig. 1b). Stresses obtained for MR fluid when squeezed approach $100-150 \mathrm{kPa}$ with relatively small displacements of the order of several millimetres. These features can be used to develop novel devices using the MR fluid technology (Bonneau and Frene, 1997; Gołdasz and Sapiński, 2011; Zhang et al., 2011).

There are only few studies exploring the squeeze mode of the MR fluid operation and the involved phenomena. In the work Horak (2013), attention is given to the effect of the displaced carrier fluid from the squeeze zone. As a result, the properties of the MR fluid will change during its operation in the squeeze mode.
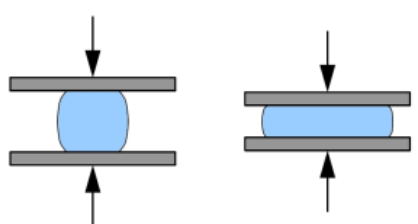

a)

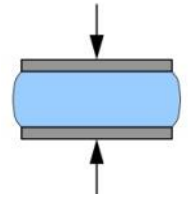

b)

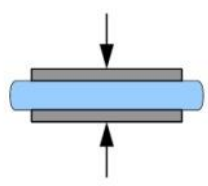

Fig. 1. Squeeze of the MR fluid: a) with the constant volume, b) with the constant contact area

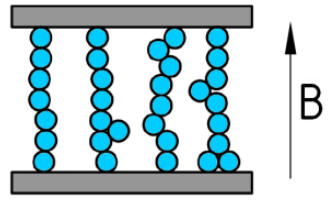

a)

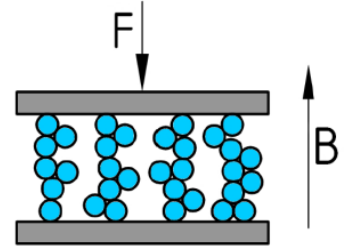

b)
Fig. 2. MR fluid particles a) column-like chain structures, b) deformation due to compression

Another phenomenon observed when a MR fluid is operated in the squeeze mode is the change of the fluid structure. In the presence of a magnetic field, ferromagnetic particles are arranged in column-like chain structures (Fig. 2a) which get deformed due to compression (Horak, 2013; Tao, 2011) (Fig. 2b). 
When operated in the squeeze mode, the MR fluid provides increased resistance to compressive loading and its yield stress tends to increase. As a result, the yield stress of the MR fluid may increase even 6-times (Tao, 2011).

Another aspect of the MR fluid behaviour in the squeeze mode is the clumping effect (Farjoud et al., 2008; 2011) which involves aggregation of MR fluid particles leading to formation of ferrous particle aggregate clumps in the fluid. As a result, progressively increasing force will develop in consecutive test runs.

Moreover squeeze force is also influenced by pressure generated in the MR fluid under the action of the applied magnetic field.

\section{EXPERIMENTS}

\subsection{Examined fluids}

Testing is done on three MR fluids, two manufactured by the BASF Company (Basonetic 2040, Basonetic 4035) and MRF-122EG, manufactured by Lord Corporation (http://www.basonetic.com/, http://www.lord.com/). The choice of those particular fluids was prompted by the intention to conduct an experiment on a MR fluid with a relatively low volumetric density $\left(<3 \mathrm{~g} / \mathrm{cm}^{3}\right)$. Similar density of tested fluids is indicative of similar solid fraction. The percentage of ferromagnetic particles in MR suspension is an important factor affecting the behaviour of the MR fluids.

MR fluids Basonetic 4035 and MRF-122Eg have a similar viscosity in the absence of the magnetic field (indicated by similar viscosity of the carrier fluid) but differ significantly in the level of saturation magnetisation. Basonetic 2040 features a higher zero-state viscosity and its saturation magnetisation level is approaching that displayed by Basonetic 4035. Selected properties of fluids used in tests are summarised in Tab. 1.

Tab. 1. Properties of MR fluids

\begin{tabular}{|l|c|c|c|}
\hline \multirow{2}{*}{ MR fluid } & Density & $\begin{array}{c}\text { Dynamic viscosity } \\
(\mathrm{B}=0[\mathrm{~T}], \hat{\gamma}=100[1 / \mathrm{s}])\end{array}$ & $\begin{array}{c}\text { Saturation } \\
\text { magnetization }\end{array}$ \\
\cline { 2 - 4 } & {$\left[\mathrm{g} / \mathrm{cm}^{3}\right]$} & {$[\mathrm{Pa} \cdot \mathrm{s}]$} & {$[\mathrm{kA} / \mathrm{m}]$} \\
\hline Basonetic 2040 & 2.47 & 0.956 & 418 \\
\hline Basonetic 4035 & 2.68 & 0.106 & 417 \\
\hline MRF-122EG & 2.38 & 0.127 & 359 \\
\hline
\end{tabular}

\subsection{Experimental set-up}

Tests were performed in the experimental set-up shown schematically in Fig. 3 (National Science Centre, contract number 1185/B/T02/2011/40). Testing device consist of a frame on which a linear servo-motor (1) is mounted, enables to change the position of the measuring plate with the accuracy $0.001 \mathrm{~mm}$. A force sensor (2) is attached to the motor, used for measuring the tensile and compression forces. The sample of the MR fluid was placed inside the test cell (3).

The investigated sample of MR fluid (5) was placed between the electromagnet cores (7) and a movable plate made of a paramagnetic material (4). The magnetic circuit is closed by the chamber housing (6). The applied magnetic field induction is altered by varying the current intensity in the electromagnet coil (8). In the electromagnet core and in the housing the ducts are made to allow the flow of the cooling fluid, which ensures the temperature stabilisation of the system. All experiments were conducted at the constant temperature $25^{\circ} \mathrm{C}$.

A PC with the dedicated software (LabView version 2010) enables the control of position and speed of the linear motor and steering of the electromagnet power supply, as well as data acquisition. a)

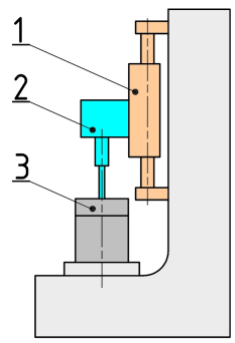

b)

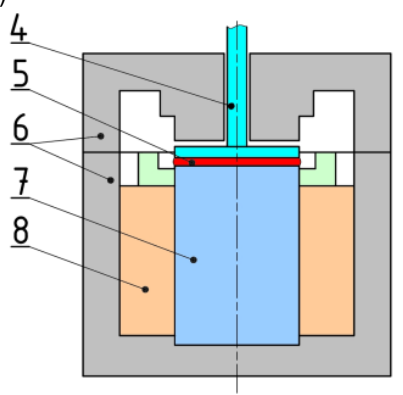

Fig. 3. Schematic diagram: a) experimental set-up, b) test cell

The schematic of the squeeze flow geometry is shown in Fig. 4. A mobile plate with the diameter $\varnothing 45 \mathrm{~mm}$ was placed between the electromagnet core and the cell housing, having identical diameter. The height of the gap between the core and the cell cover is $h_{g}=10 \mathrm{~mm}$, the sample volume $1.6 \mathrm{ml}$ and the initial height of the gap is $h_{0}=1 \mathrm{~mm}$. The amplitude of the applied triangular excitation inducing the plate position is $\Delta \mathrm{h}=0.2 \mathrm{~mm}$.

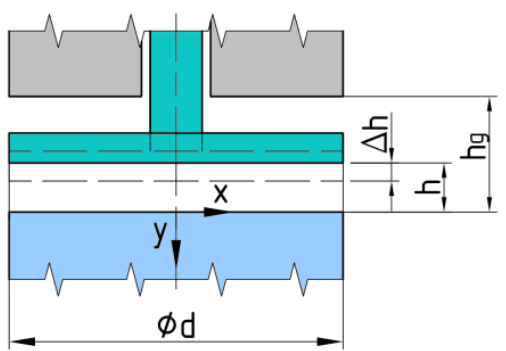

Fig. 4. Schematic of geometry

Experiments were performed in the presence of the magnetic field with the flux density B: 70, 100, 140, 200 and 270 [mT] under the applied excitation with the frequency $\mathrm{f}=0.1$ and $0.5[\mathrm{~Hz}]$, corresponding to the squeeze rate 0.04 and $0.02[\mathrm{~mm} / \mathrm{s}]$. The squeeze force was assumed to have the positive sign.

\subsection{Results}

Fig. 5 shows plots of squeeze force and of the applied excitation (changes in the plate's position) for one of the MR fluids (MRF-122EG) and for various levels of magnetic flux density. The plots of displacement excitations for $\mathrm{f}=0.1$ and $0.5 \mathrm{~Hz}$ are slightly different, which is associated with the accuracy of level involved in the linear motor executing the pre-set operation pattern.

The relationship between the squeeze force $(F)$ and change of the gap height $(\Delta h)$ is shown in Fig. 5, 6 and 7. For all investigated fluids, during the return movement of the plate (gap increase), the squeeze force rapidly decreases, going down to its initial level. 
a)

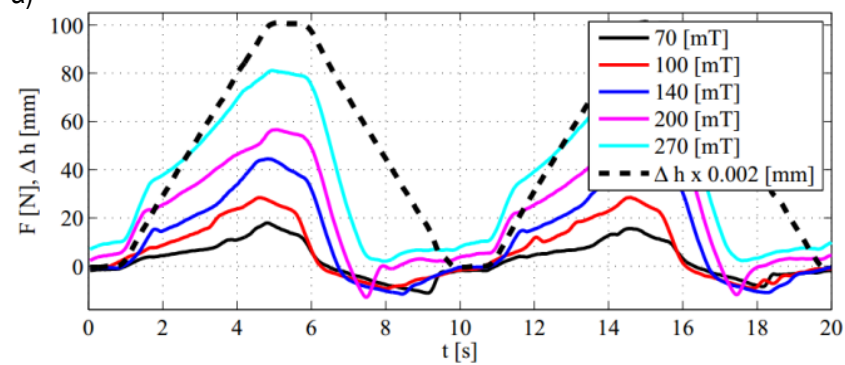

b)

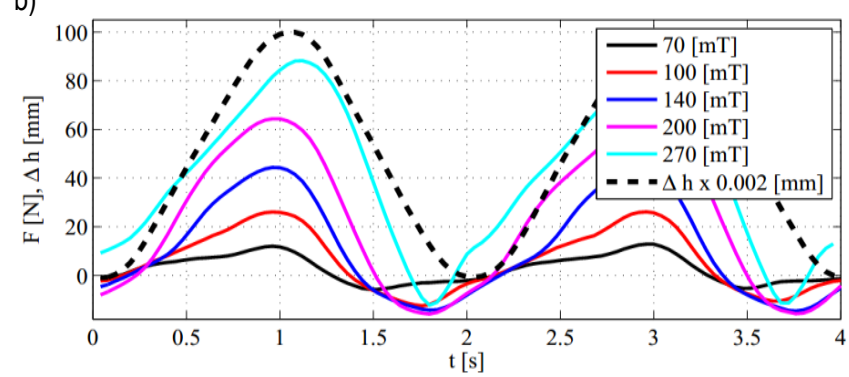

Fig. 5. Squeeze force and plate displacement vs. time; MRF-122EG;

a) $f=0.1 \mathrm{~Hz}$, b) $f=0.5 \mathrm{~Hz}$

For flux density $B<100 \mathrm{mT}$, the force increase is linear for the fluids Basonetic 4030 and MRF-122EG. In the case of the fluid Basonetic 2040, however, a dramatic force increase was registered in the final phase of the squeezing process.

For flux density $B>140 \mathrm{mT}$, a two- stage force increase was observed for MRF-122EG and Basonetic 4035. In the first stage the squeeze force increases rapidly and then begins to increase linearly, at a lower rate, finally reaching its maximal value. Revealed differences between force vs. gap height plots obtained for MR fluids differing in viscosity of their carrier fluids may be indicative of the influence that the structure of the fluid has on the process of squeezing. In Basonetic 2040 (based on a highviscosity oil), formation of chain structures is hindered because of higher resistance to motion experienced by ferromagnetic particles. In the case of various MR fluids subjected to magnetic field with the same flux density, those with less extended particles structures will experience squeezing.

For the fluid featuring the highest zero-state viscosity (Basonetic 2040), the squeeze frequency does not significantly affect the registered force pattern. The influence of the squeeze rate on the squeeze force is revealed only in fluids whose carrier fluid has lower viscosity (MRF-122EG and Basonetic 4035). This behaviour of MR fluids may be indicative of the influence that the rate of the chain structure formation has on the squeezing force. A high squeeze frequency shortens the time needed by the MR fluid particles to form a chain structure. In MR fluids containing low-viscosity carrier fluids formation of chain structures will take less time.

For flux density $B=270 \mathrm{mT}$, a non-zero force is registered whilst the moving plate returns to its initial position. This force is the consequence of pressure developed in the MR fluid due to the action of a magnetic field. A thorough analysis of this phenomenon is presented in the work Salwiński and Horak (2012).

The plots of maximal squeezing force are provided in Fig. 9. No matter what the squeezing rate, for lower flux densities the greatest resistance to squeezing is registered for fluids with highest viscosity. This may by cause to the fact that under the action of weaker fields, the carrier fluid viscosity becomes the major determinant of the MR fluid behaviour. High viscosity of the carrier fluid may be responsible for lower values of the maximal squeeze force registered in measurements taken at higher flux densities.
Most probably, high viscosity of the carrier fluid impacts on the dynamics of MR particles forming the chain structures, that is why at the instant the squeezing begins, the less developed particle structure will get deformed.

a)

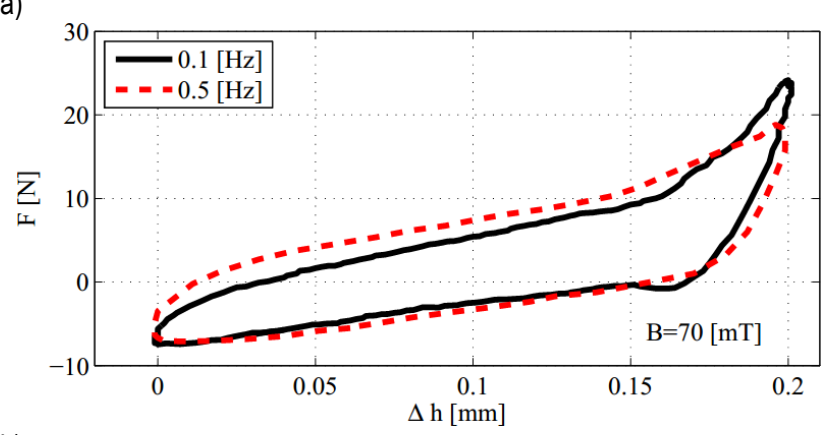

b)

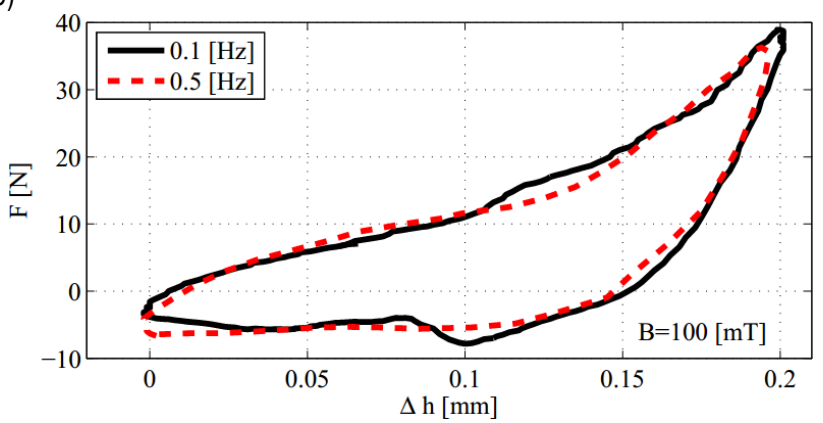

c)

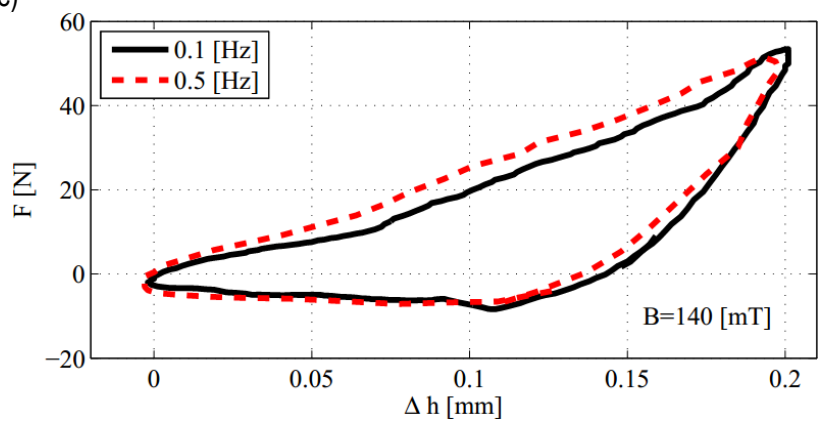

d)

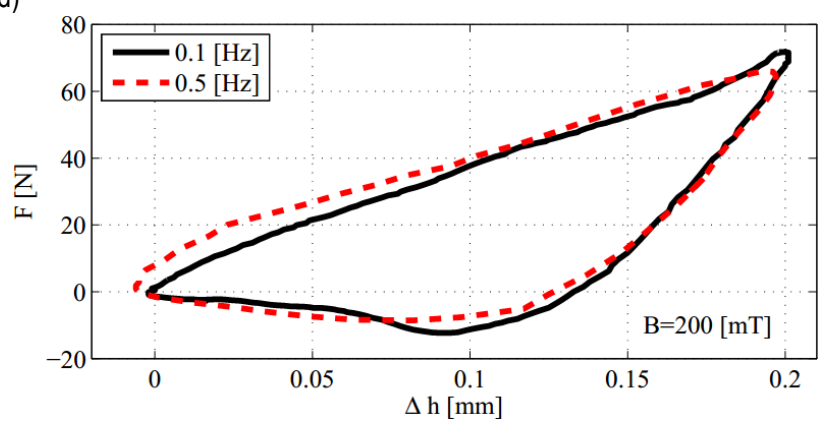

e)

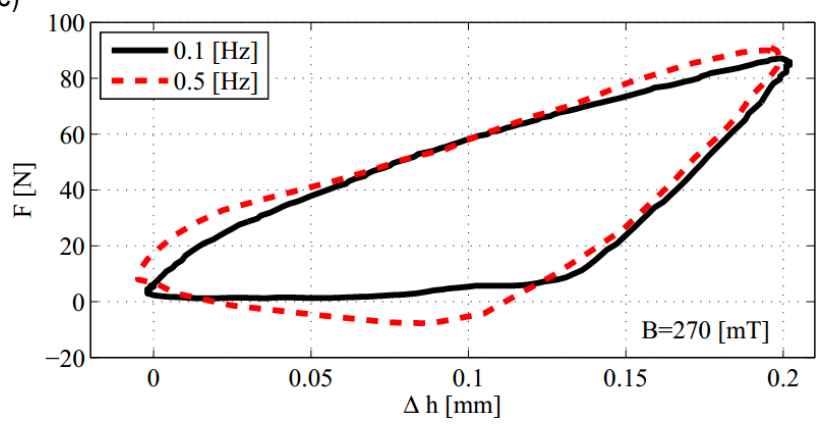

Fig. 6. Force vs. time; Basonetic 2040 
Bogdan Sapiński, Wojciech Horak,Marcin Szczęch

a)

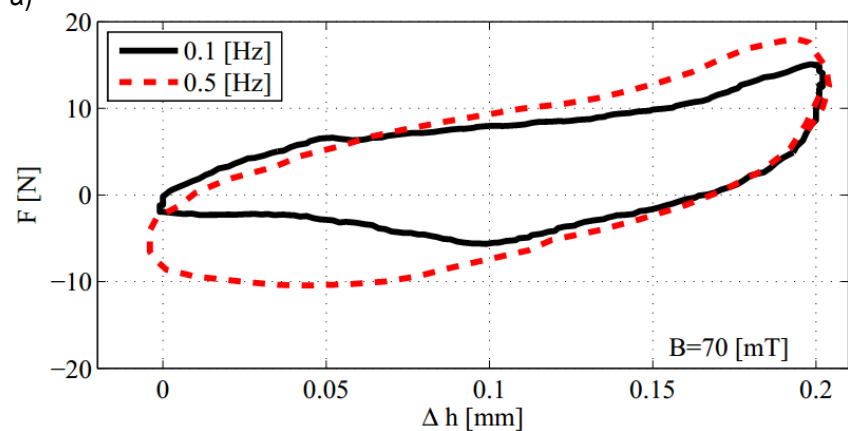

b)

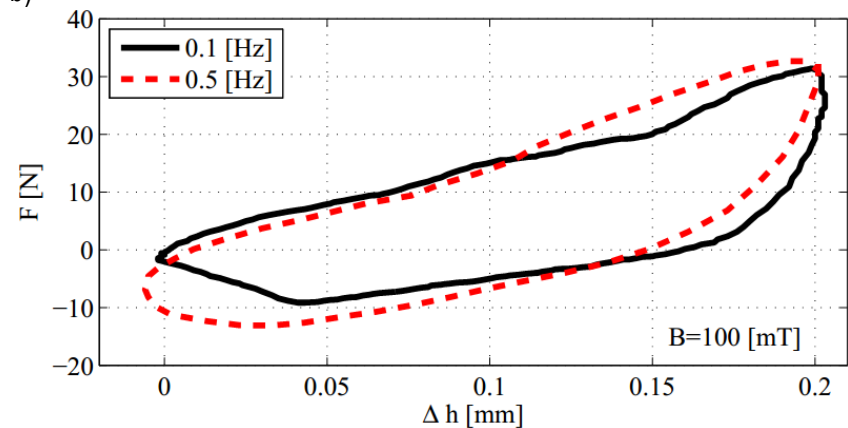

c)

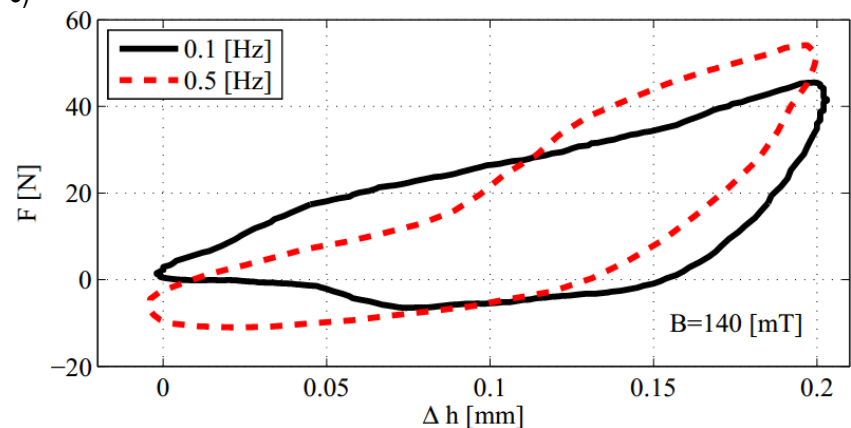

d)

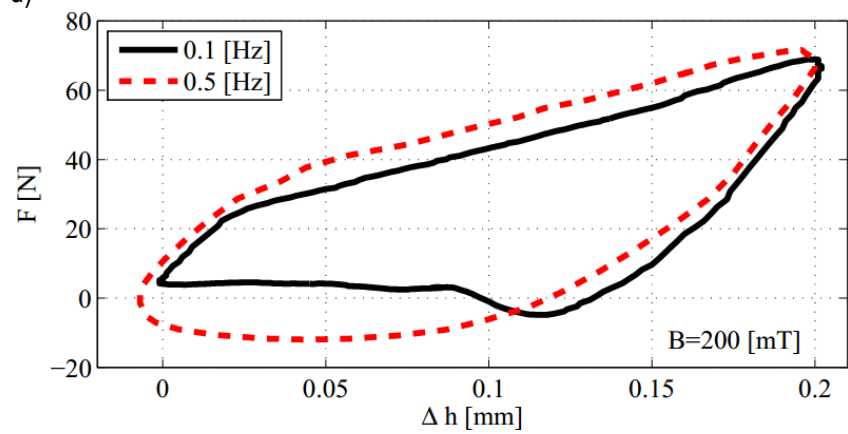

e)

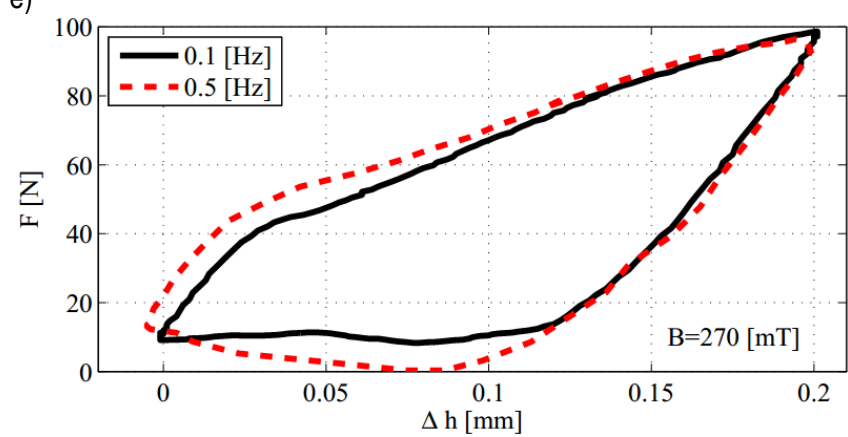

Fig. 7. Force vs. time; Basonetic 4035 a)

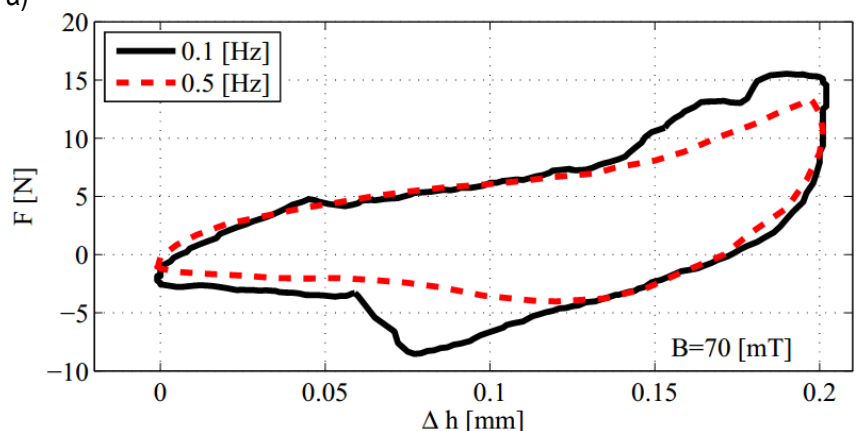

b)

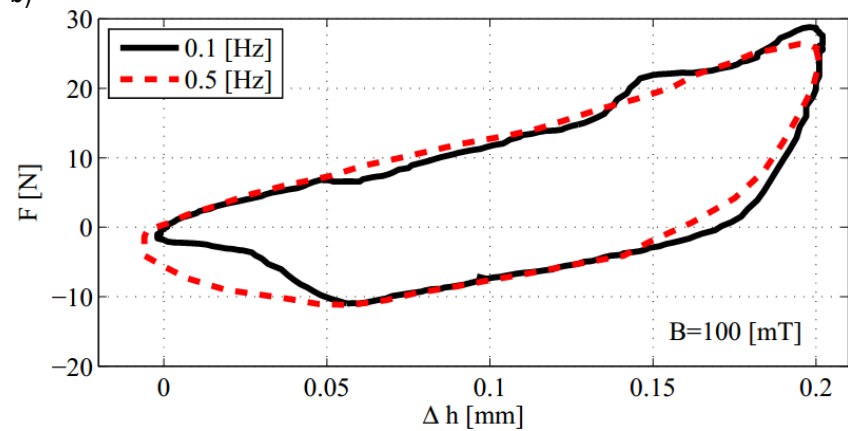

c)

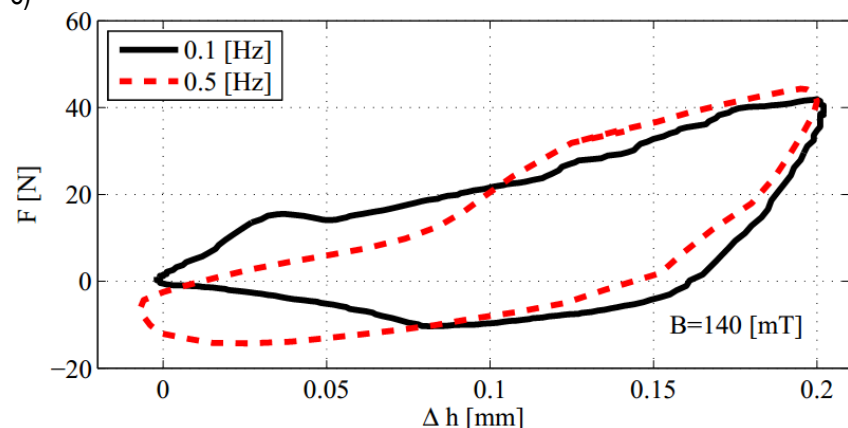

d)

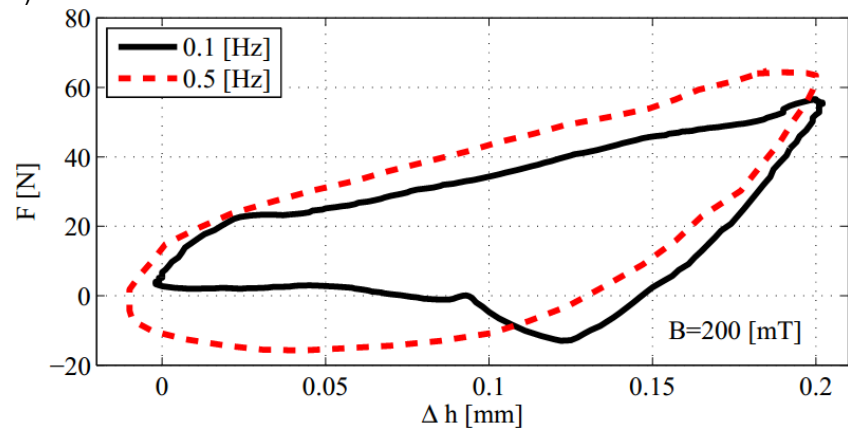

e)

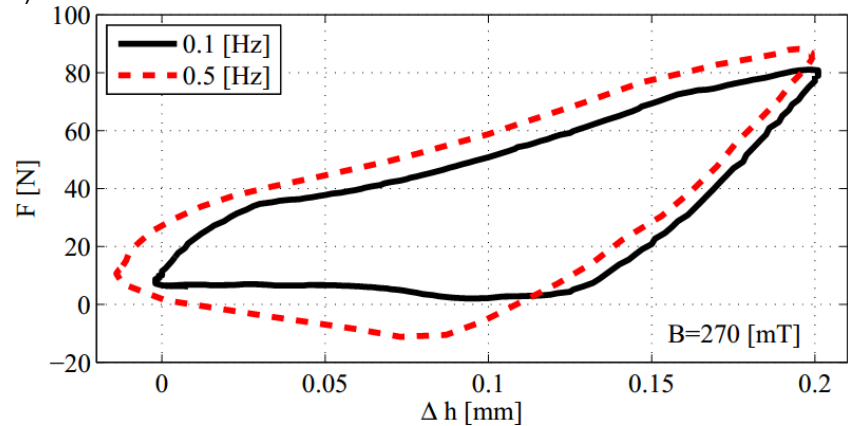

Fig. 8. Force vs. time; MRF-122EG 
a)

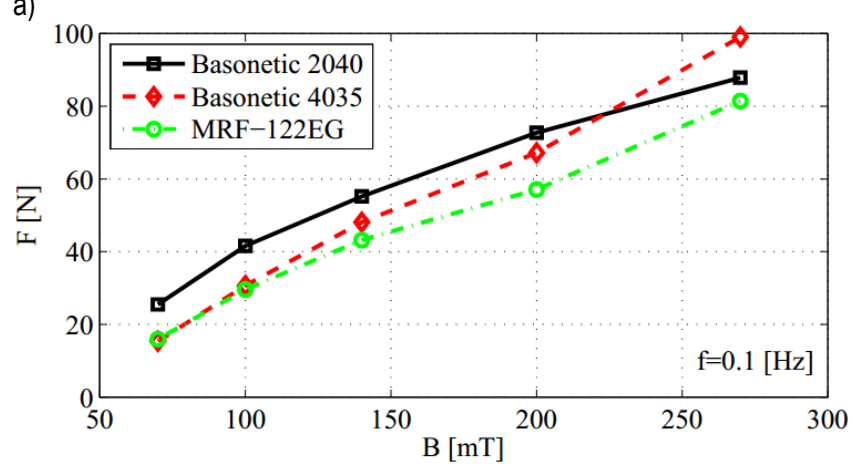

b)

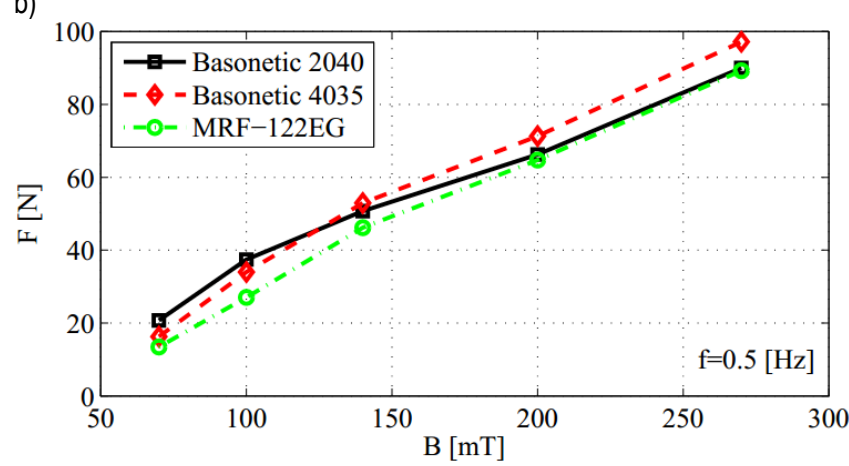

Fig. 9. Maximal force vs. flux density

It is worthwhile to mention, that for flux densities $B<100 \mathrm{mT}$ the maximal squeezing forces registered for MR fluids with similar viscosity yet differing in saturation magnetisation are similar, evidencing the dominant influence that viscosity of the carrier fluid has on the behaviour of MR fluids operated in the squeeze mode under the action of magnetic field with a relatively low flux density.

The highest value of the maximal squeezing force is obtained for the MR fluid featuring high saturation magnetisation and low viscosity. The squeezing rate is found to have little effect on maximal squeeze force.

Fig. 10 shows the photo of MR fluid (MRF-122EG) after the experiments, revealing the carrier fluid released from the MR fluid. The identical process is observed for all investigated fluids. Release of the carrier fluid is associated with the fact that ferromagnetic particles in the squeeze zone are maintained in position by the magnetic field and the carrier fluid mostly will be pushed from the gap, causing the rheological properties of MR fluids to be altered. That is so because the (percentage) proportion of the solid phase in MR fluid is changed, too.

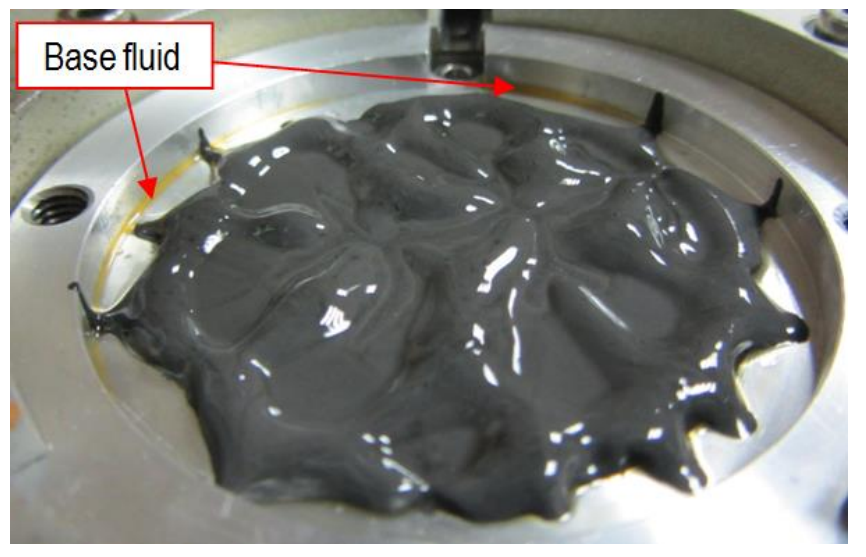

Fig. 10. MRF-122EG after experiments

\section{SUMMARY}

The paper summarises the results of testing of three commercially available MR fluids (Basonetic 2040, Basonetic 4035, MRF$122 E G)$ operated in the oscillatory squeeze mode. Investigated fluids differed in their zero-state viscosity and in the level of saturation magnetisation.

The resistance to squeeze exhibited by MR fluids are determined by several factors, such as: yield stress, viscosity, strength of chain-like structures of ferromagnetic particles, the rate of formation of chain structures. Furthermore, the magnetostatic pressure associated with MR fluid and magnetic field interactions is a major determinant, too.

The behaviour of all investigated MR fluids was similar. The differences between the registered force values and the shapes of the squeeze force plots are attributable to various levels of intricacy of MR fluid particle structures and differences in viscosity of carrier fluids in the investigated samples.

It was observed that the carrier fluid tends to get separated from the ferromagnetic particles during the experiments.

No progressively increasing squeeze force was observed in consecutive squeeze test runs. It is reasonable to suppose, therefore, that no 'clumping effect' occurred during the experiments.

The behaviour of MR fluid operated in the squeeze mode is a complex research problem. At the current stage it is impossible to address and explain all aspects involved in the squeeze mode of MR fluid operation. The analysis is further complicated by lack of specific data about the composition and structure of investigated fluids, for instance the shape and size of forming particles.

\section{REFERENCES}

1. Bonneau O., Frene J. (1997), Non-linear behavior of a flexible shaft partly supported by a squeeze film damper, Wear, 206, 244- 250.

2. Chengye L., Fengyan Y., Kejun J. (2011), Design and finite element analysis of magnetic circuit for disk MRF brake, Advanced Materials Research, 181-182, 22-527.

3. Farjoud A., Craft M., Burke W., Ahmadian M. (2011), Experimental investigation of MR squeeze mounts, Journal of Intelligent Material Systems and Structures, 22, 1645-1652.

4. Farjoud A., Vahdati N., Fah Y. (2008), MR-fluid yield surface determination in disc-type MR rotary brakes, Smart Materials and Structures, 17, 3, 1-8.

5. Gołdasz J., Sapiński B. (2011), Model of a squeeze mode magnetorheological mount, Solid State Phenomena, 177, 116-124.

6. Gołdasz J., Sapiński B. (2011), Modelling of magnetorheological mounts in various operation modes, Acta Mechanica et Automatica, 5, 29-40.

7. Guldbakke J. M., Hesselbach, J. (2006), Development of bearings and a damper based on magnetically controllable fluids, Journal of Physics: Condensed Matter, 18, 38, 2959-2972.

8. Horak W. (2013), Theoretical and experimental analysis of magnetorheological fluid squeeze flow mode, PhD Thesis, AGH University of Science and Technology, Krakow.

9. Mazlan S. (2008), The behaviour of magnetorheological fluids in squeeze mode, PhD Thesis, Dublin City University.

10. Salwiński J. Horak W. (2012), Measurement of normal force in magnetorheological and ferrofluid lubricated bearings, Key Engineering Material, Vol.490, 25-32.

11. Sapiński B. (2006), Magnetorheological dampers in vibration control, Uczelniane Wydawnictwa Naukowo-Dydaktyczne AGH, Kraków.

12. Tao R. (2011), Super-strong magnetorheological fluids, Journal of Physics: Condensed Matter, 13, 50, 979-999. 
13. Zhang X. J., Farjud A., Ahmadian M., Guo K. H., Craft M. (2011), Dynamic Testing and Modelling of an MR Squezee Mount, Journal of Intelligent Material Systems and Structures, Vol. 22, 1717-1728.

14. BASF The Chemical Company, http://www.basonetic.com/

15. LORD Corporation, http://www.lord.com/

16. Theoretical and experimental analysis of thrust slide bearing lubricated with magnetic fluid. National Science Centre, contract number 1185/B/T02/2011/40.

This research is supported by the National Centre for Research and Development under grant No. PBS 1/A6/3/2012. 